\title{
etapas en el desarrollo del control de hornos de Eemento por medio de computadores
}

\section{INTRODUCCION}

1) El repasar la experiencia adquirida y que culminó en el empleo de sistemas de control por computador en una de las fábricas del Reino Unido, puede ser de interés tanto a aquellos que piensan adoptarlo como aquellos otros que ya lo han hecho. Sería quizás mejor empezar por decir algo sobre nuestra empresa y su relación con la industria del cemento británica. La demanda de cemento en el país es de unos 17 millones de toneladas anuales, y la participación de nuestra compañía es del orden del $14 \%$. Tenemos tres fábricas: dos en el sudeste y una en el noroeste de Inglaterra. La mayor está en West Thurrock y tiene una producción anual de 1,2 millones de toneladas. Es en ella en la que estamos centrando nuestra actividad actual en relación con el control por computador. Los ocho hornos de esta fábrica funcionan por vía húmeda. Las materias primas

son una caliza blanda y una arcilla de Londres. La pasta arcillosa se prepara a cierta distancia y se bombea a los desleidores de caliza en la fábrica para preparar una pasta. De vez en cuando surgen variaciones en la calidad de la pasta, y éstas se reducen por homogeneización en una serie de vasos y también por medio de molinos tubulares y una instalación trix. La pasta homogeneizada se alimenta directamente a los hornos.

2) Los ocho hornos son de distintas capacidades y edades y pueden dividirse en dos grupos principales: uno de ellos tiene una producción de $300 \mathrm{t} /$ día y el otro de 600 a 700 t/día. Hay diferencias en el diseño de los hornos, pero todos son de vía húmeda y están en su mayoría equipados con enfriadores planetarios, teniendo dos de ellos enfriadores de parrilla. En la fábrica hay varios molinos de cemento de distintas capacidades, y la mayor parte del cemento se hace en las calidades de portland ordinario o de endurecimiento rápido.

\section{ESTUdio de los Metodos de CONTRol del PROCESo}

3) El proyecto que ha conducido a la instalación de un computador de control de procesos se inició hace unos 2 años con un estudio de los procedimientos de control que se empleaban en las fábricas. Esta revisión no se limitó al departamento del horno, sino que también incluyó los siguientes departamentos:

i) materias primas;

ii) preparación de pasta;

iii) molienda de cemento. 
4) Los objetivos de este estudio de los procedimientos de control fueron los siguientes:

i) Descubrir las áreas, si las hay, en las cuales puedan hacerse mejoras en el control del proceso. Se pensó que se podrían conseguir mejoras en varios aspectos:

- mayor producción;

b mejor uso del combustible y de la energía;

c mejora del control de calidad;

d menor costo de fabricación;

e mayores beneficios.

ii) Habiendo establecido las áreas donde se pueden hacer mejoras, proponer proyectos de desarrollo por un sistema de etapas enumerando las ventajas económicas que justifiquen el adquirir un sistema importante de control. En ese caso se sugirieron un cierto número de procedimientos de control mejorados que ahora se están poniendo en práctica. No todos ellos han necesitado el comprar material caro, y muchos de ellos se han basado en una valoración más objetiva de la información que ya se tenía, junto con una mejora de la frecuencia de muestreo.

iii) Era evidente que se tardaría bastante tiempo en trazar en toda la fábrica un esquema de control avanzado del tamaño y complejidad que estamos discutiendo. Si estos esquemas avanzados incluyesen el empleo de utillaje complejo (por ejemplo computadoras para controlar procesos y técnicas analíticas on-line), pasaría bastante tiempo entre la decisión de comprar el utillaje y sú instalación y ponerlo a punto en todas las instalaciones de la fábrica.

5) Así, de ser posible el proceso de desarrollo, debe planearse de modo que las unidades que estén en una fase más avanzada de mejoramiento del sistema de control puedan beneficiarse de sistemas mejorados de control manual, basados en resultados de la investigación, que conduzca a la instalación de sistemas más complejos.

\section{ETAPAS EN EL DESARROLLO DEL SISTEMA DE CONTROL}

6) Los argumentos anteriores han llevado al desarrollo de un sistema mejorado del control de los hornos por etapas, y estas etapas pueden identificarse como:

i) control manual mejorado del horno;

ii) sistema de control inicial del horno por computador basado en una serie de bucles cerrados individuales de control, junto con algoritmos de control más complejos para guiar los puntos fijos de los controles individuales;

iii) desarrollos en relación con ii), que han tenido lugar en dos direcciones:

a un mayor desarrollo de control algorítmico basado en estudios estadísticos de relación entre fábricas;

b desarrollo de modelos teóricos del horno. 
7) De hecho se ha encontrado que es conveniente hacer uso de los resultados de estos estudios para mejorar los procedimientos de control manual. En todas las etapas fue necesario calcular las ventajas económicas antes de proseguir a la etapa siguiente.

\section{RESULTADOS DE UN ESTUDIO INICIAL DE LOS PROCEDIMIENTOS DE CONTROL}

8) Aunque el estudio incluyó todas las áreas de la fabricación, este trabajo se centra. en el departamento del horno para ilustrar las técnicas adoptadas.

9) El análisis del sistema manual utilizado para controlar el horno, señala la posibilidad de que la capacidad y la eficacia del horno estén limitadas por el propio sistema del control mismo. En particular, se examinó el procedimiento empleado por los operadores de la fábrica para cerrar uno de los bucles principales de control del horno: la valoración visual de las condiciones en la zona de clinkerización, seguida de un ajuste por parte del operario del combustible que entra al horno. Se decidió que si se pudiera encontrar una regla sencilla y objetiva para ayudar al operador a tomar una decisión correcta en el control, se eliminarían muchas operaciones erráticas. Además, al obtener una ventaja inmediata reduciendo el tiempo que se produce material mal cocido y al lograr velocidades de producción media más elevadas, estas mejoras facilitarían también la tarea de entender mejor las relaciones interiores del horno, en caso de que el desarrollo posterior indicase la conveniencia de construir algún modelo estadístico de horno. En este aspecto se consideró que sería más rápido establecer un modelo estadístico. Se apreciaron las ventajas y desventajas de modelos tanto estadísticos como teóricos y se decidió desarrollar los dos tipos de modelo.

\section{PROCEDIMIENTO ORIGINAL DE CONTROL DEL FUEL-OIL}

10) Una de las principales funciones del operador es determinar la cantidad correcta de fuel-oil para unas condiciones de entrada fijas del horno.

La cantidad de fuel-oil se determinó, principalmente, por:

i) velocidad de alimentación de pasta;

ii) velocidad del horno;

iii) contenido de agua en la pasta;

iv) aptitud a la cocción de la pasta.

En el procedimiento original, el operador hacía un cálculo visual de las condiciones en la zona de cocción, incluyendo temperatura y profundidad del lecho de material sólido, y de acuerdo con esto ajustaba la válvula del combustible. El operador también tenía en cuenta otras condiciones: consumo de energía del horno, condiciones de trabajo del enfriador, contenido de oxígeno de los gases de escape, posición del registro de tiro, y temperatura a la salida del horno; así como el ajuste de la forma de la llama. El operador contaba con pocos medios para valorar su propia competencia de un modo continuo y esto conducía a considerables variaciones en el control correcto en cada momento.

11) Empleando estas técnicas, y porque esencialmente se estaba intentando equilibrar dos cantidades de calor grandes sin valorar objetivamente, el operador, con mucha frecuencia, realizaba un ajuste incorrecto de válvulas: si el valor era muy alto, el mate- 
rial en la zona de cocción se aproximaría demasiado a su temperatura de fusión. Entonces, tendería a formarse un anillo que, más pronto o más tarde, habría que romper causando un aumento rápido en el flujo de material (disminución del tiempo de permanencia) a través de la zona de sinterización. El retraso en apercibirse de este hecho el operador puede ser tal, que él se vea imposibilitado de enfrentarse eficazmente con el aumento rápido del flujo de material y, con ello, que se produzca un clínker de calidad anormal. Frecuentemente es necesario reducir la velocidad de producción hasta volver a conseguir condiciones estables de control.

12) Se realizaron ensayos para justificar la explicación anterior del ciclo de control: Un ejemplo de las investigaciones de los cambios en el flujo de material, en el flujo de fuel-oil, en el peso por litro y en la cal libre está indicada en las figuras 1a y $1 \mathrm{~b}$, que demuestran la correlación entre estas variables e indican asimismo la dificultad de control con que se enfrenta el hornero.

13) Los valores de la temperatura en la zona de cocción también habrían sido útiles como ayuda para interpretar los datos, pero el horno no tenía un pirómetro adecuado en aquella época.

\section{CONTROL MANUAL MEJORADO}

14) Parece que el sistema de control manual podría mejorarse si el hornero dispusiera de una válvula de punto fijo para el suministro del fuel-oil. Aún en el caso de que esto supusiera unas condiciones estacionarias, se cree que sería una ventaja considerable. Además se pensó que podían eliminarse las perturbaciones que tienen ciclos de tiempo cortos y que se dęben a la alimentación de pasta quedando sólo aquéllas que tienen ciclos de tiempo más largos. Estas últimas pueden corregirse empleando un sistema de corrección de tendencia basado en el análisis de las propiedades del clínker (por ejemplo peso-litro o cal libre).

15) Se ha dedicado bastante esfuerzo a mejorar el control de la alimentación de pasta, y esto está ahora dando buen resultado. En este trabajo no se intentará describir este aspecto del problema.

\section{ESTIMACION DEL VALOR CONSIGNA DEL FUEL-OIL}

16) Al estimar el valor consigna, se enfrenta uno con un dilema. Idealmente este valor debería calcularse a partir de modelos del proceso. Se sabe, por consideraciones teóricas, que para mejorar el control por este procedimiento habría que calcular con bastante precisión dicho valor consigna (por ejemplo 30 galones/hora). Esto parece que elimina los modelos teóricos en esta fase si se ha conseguido una velocidad de desarrollo razonable. A primera vista parece indicado hacer uso de un modelo estadístico, pero ello requeriría una marcha más estable de la fábrica de lo que actualmente se puede conseguir. La táctica adoptada ha sido la de emplear nomogramas Shewhart y nomogramas de suma acumulativa para analizar la historia reciente de la fábrica. Los operadores recibieron instrucciones para la utilización de estos nomogramas, y trabajando inicialmente con una estricta supervisión emplearon la información para adoptar acciones de control adecuadas. Las variables de la fábrica que se establecieron por este medio fueron las siguientes:

i) velocidad de flujo de fuel-oil (cantidad horaria totalizada);

ii) carga del horno, en amperios;

iii) peso-litro (medido cada hora). 


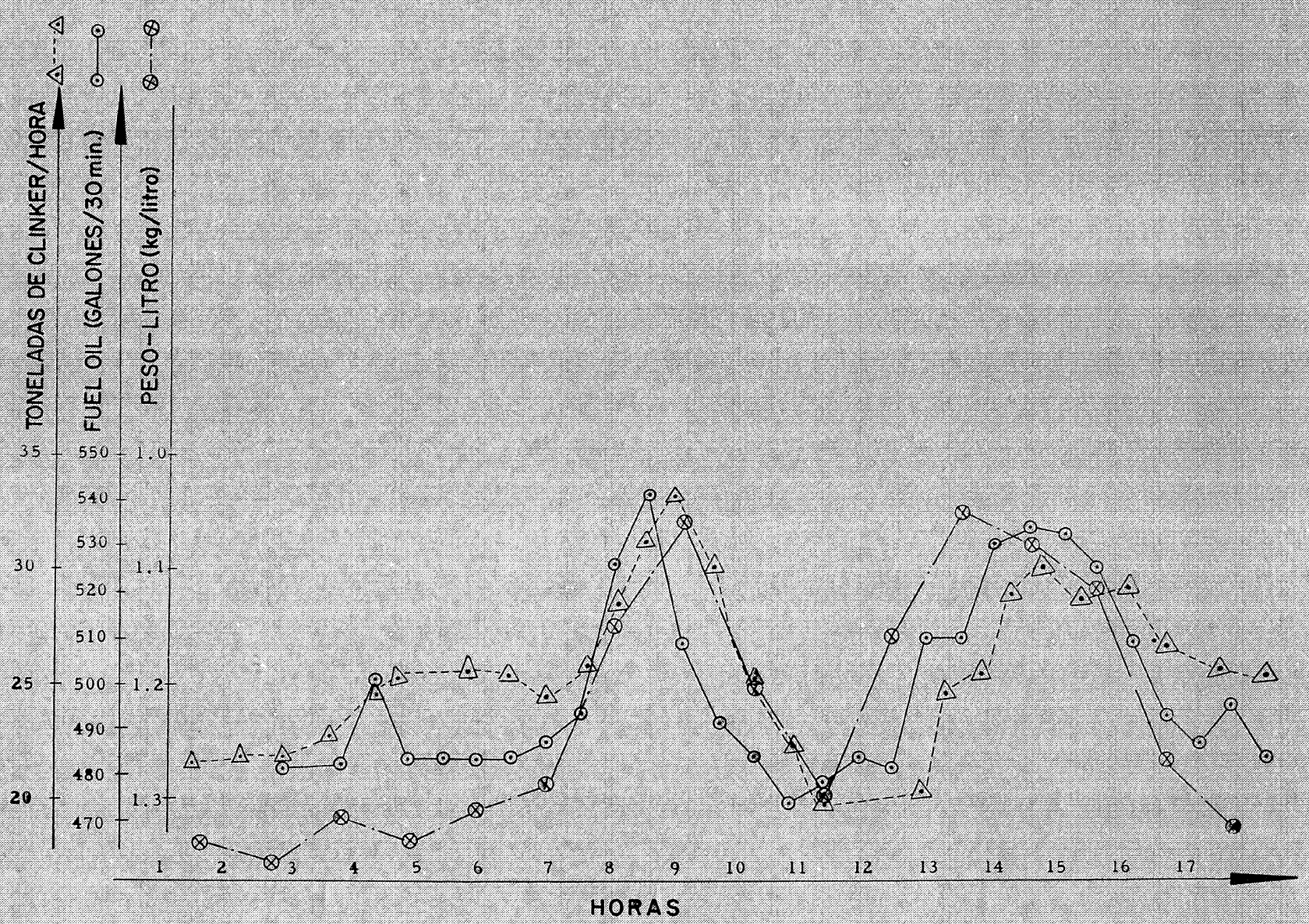

Fig. 1a.-Relación entre las variables del horno en ensayos de control manual.

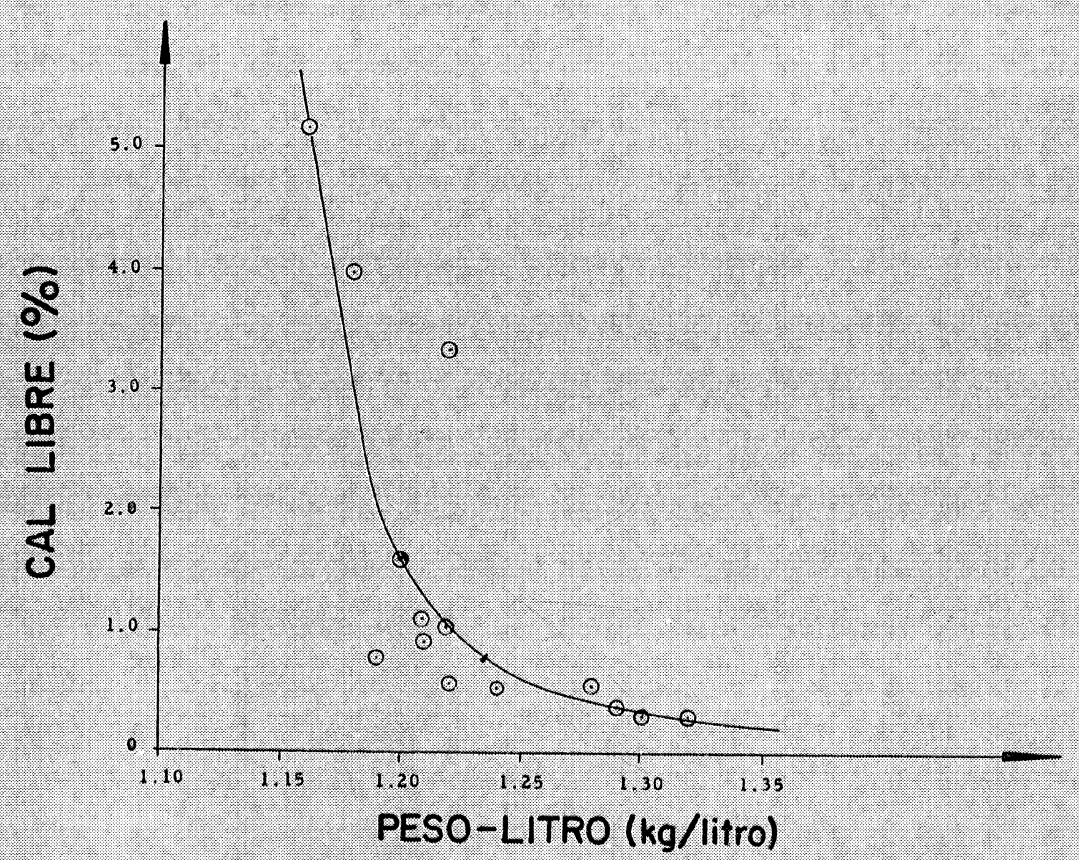

Fig. 1b.-Ensayos de control manual. Relación entre la cal libre y el peso-litro. 
17) El valor consigna del fuel-oil se calculó examinando resultados de los nomogramas en un período previo de 6 a 10 horas. Siempre que no haya cambio en la velocidad de alimentación de pasta, en el contenido de agua de la misma o en la velocidad del horno, puede emplearse una primera estimación del valor consigna del fuel-oil comprendida entre 20 y 30 galones del valor medio estimado. La acción de control que se puede tomar depende de la magnitud de la diferencia entre el valor actual del fuel-oil y el valor consigna que se ha establecido. Otra acción de control puede tomarse basada en la tendencia del análisis del peso-litro registrado. Esta debe considerarse comó una corrección secundaria, ya que la acción de control no se toma hasta que no existe una tendencia estadística apreciable en el peso-litro.

18) Este sistema manual se ha ensayado con éxito en varios hornos, con resultados prometedores. El valor del sistema puede apreciarse en la figura 2, que muestra algunos aspectos del control de horno antes y después de introducir el sistema. El control mejorado ha permitido una mayor producción. Aunque no se demuestra, en esta etapa también se consiguieron mejoras en la economía de combustibles.

19) Los ensayos iniciales demostraron que ese procedimiento elimina muchas de las variaciones debidas al sistema de control inicial. Después era posible realizar una serie de ensayos en la fábrica proyectados estadísticamente, con objeto de establecer las relaciones entre las distintas variables que rigen el proceso del horno.

\section{APROVECHAMIENTO DEL HORNO}

20) El aprovechamiento de un horno se obtiene al tener en cuenta factores tales como velocidad de flujo de la pasta en el horno, contenido de agua de la misma y veloci-

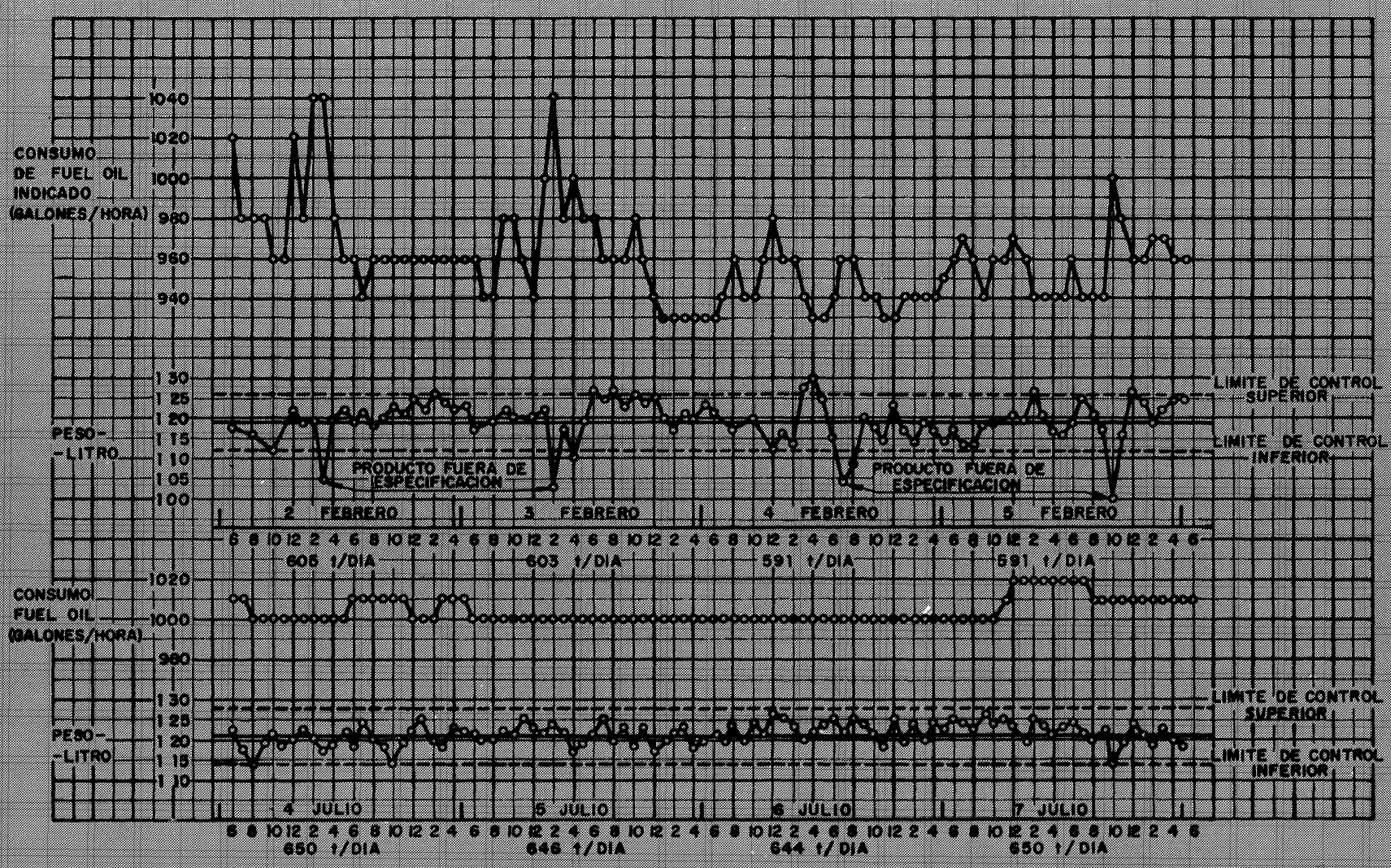

F15. 2.- Brecto del procedimiento de control manual ineforado. 
dad de rotación del horno, junto con una serie de condiciones que deciden la eficacia del combustible. La táctica adoptada en el desarrollo de modelos de fábrica era establecer el efecto de pequeñas variaciones con relación a los niveles normalmente aceptados de las variables que actúan en la eficiencia de la planta, los costos de operación y el aprovechamiento. Una vez que se han establecido una serie de condiciones de funcionamiento que son mejores, se toman éstas como las nuevas condiciones standard y se ensaya un nuevo conjunto de variaciones.

21) Durante la realización de esta táctica se llega a un estado en que ya se han estudiado amplias zonas de algunas de las variables de la operación, siendo entonces posible analizar los resultados de estos ensayos en su conjunto y, por consiguiente, se pueden desarrollar los modelos del proceso.

Los costos fijos y variables del horno son tales que el aprovechamiento depende, sobre todo, de aumentar al máximo la producción y la eficacia del combustible. Se ha decidido concentrar el estudio inicialmente en un intento de llevar al máximo la producción; las variables que se han empleado en los primeros ensayos han sido las siguientes:

i) velocidad de flujo de la pasta;

ii) contenido de agua de la pasta;

iii) velocidad del horno.

Durante esta fase de desarrollo se obtuvo una fórmula para ajustar el flujo de aire primario y secundario y conseguir así condiciones satisfactorias de cocción por cambios en otras variables de la fábrica, lo cual se logró en colaboración con todo el personal técnico. Aunque inicialmente el combustible no se emplee de la manera más eficaz se ha pensado que este aspecto puede estudiarse más adelante, una vez que se han conseguido los valores más altos de producción. De hecho, durante los ensayos, se logró una mejora en eficacia del combustible de un $5 \%$ y se mejoró la producción en un horno en $10 \%$ por lo menos. Esto ha justificado ampliamente el costo de este programa y ha sido un verdadero incentivo pasa pasar a la siguiente fase de desarrollo, es decir, estudiar el aspecto económico de sistemas más complejos de control.

22) Como resultado de los ensayos, se han desarrollado relaciones entre i) velocidad de flujo del combustible, ii) costo del fuel-oil, y iii) aprovechamiento en función de las variables dependientes e independientes dadas anteriormente. Estas relaciones se han conseguido aplicando análisis regresivos a la información hallada en los experimentos. Los resultados que encajaban mejor se obtuvieron con expresiones de términos de primer orden. Términos de segundo orden y términos mixtos no eran significativos. Esto puede ser debido a que el espacio experimental estaba limitado principalmente por las características físicas de la fábrica. Por ello, las relaciones eran todas del tipo:

$$
y=a_{1} x_{1}+a_{2} x_{2}+a_{3} x_{3}+a_{4},
$$

donde:

$y=$ variable dependiente:

$x_{1}=f$ (velocidad de alimentación de la pasta);

$x_{2}=$ contenido de agua de la pasta;

$x_{3}=$ velocidad de rotación del horno. 


\section{ARGUMENTOS ECONOMICOS EN FAVOR DE UN CONTROL POR COMPUTADOR}

23) Las relaciones del tipo indicado arriba se emplean en la fábrica para calcular el punto fijo del bucle de control del fuel-oil; la técnica de operación evolutiva se está aplicando para optimizar la producción del horno, y se utilizará para investigar el rendimiento máximo del combustible. Sin embargo, la experiencia ha demostrado que, en ausencia de una supervisión continua, los operadores de la fábrica no conseguirán condiciones de operación óptima de modo continuo. Consideradas las limitaciones del sistema manual, parece que será ventajosa alguna forma de automatización. La comparación del rendimiento de una fábrica con y sin supervisión de alto nivel demostró que el automatizar la fábrica conducirá a nuevas mejoras en el rendimiento de ésta y en el consumo de combustible, de tal modo que un sistema de control on-line por computador capaz de controlar seis hornos, y en consecuencia las fábricas mayores de cemento, debería amortizarse en menos de 2 años de funcionamiento eficaz.

La viabilidad técnica del procedimiento para calcular el punto fijo del fuel-oil quedó demostrada al analizar los datos empleando el computador ADP de la empresa, situado en su oficina central. La información se hizo pasar de la fábrica por teléfono y se perforó en la oficina central. Se calcularon los cambios en el punto fijo y se volvieron a enviar a la fábrica por teléfono. Se realizaron dos ensayos con éxito, cubriendo unas 70 horas de funcionamiento.

De estos estudios se infiere que:

i) había justificación económica para adoptar el control por computador;

ii) se podía desarrollar un posible sistema por computador.

\section{SELECCION DEL COMPUTADOR}

24) Siguió bastante trabajo para seleccionar un fabricante del computador:

i) diseño detallado del sistema de control y desarrcllo de las especificaciones del utillaje y de los medios auxiliares;

ii) revisión de la instrumentación de la fábrica y de su susceptibilidad a un control por computador;

iii) examen de propuestas y selección final del equipo.

Después de mucha discusión, se decidió comprar un computador Honeywell H.21. El computador se entregó en la fábrica en septiembre de 1967, y ahora está sometido a las pruebas finales de recepción.

\section{ESTADO ACTUAL DEL CONTROL POR COMPUTADOR}

25) Se ha visto que el computador controla satisfactoriamente algunos de los diez bucles DDC, en la forma de regulación, con puntos fijos para los bucles, insertados manualmente. El paso siguiente en el desarrollo del sistema es intentar el ajuste automático de los valores consigna, a partir de dos algoritmos de control básicos, que representan un modelo sencillo del horno. El primero de estos algoritmos calcula el consumo básico del fuel-oil en los casos en que haya variaciones en la alimentación del horno (es decir, contenido de agua en la pasta, velocidad de entrada de la pasta). Los cambios previstos en el 
suministro de fuel-oil no se aplican inmediatamente sino que se realizan por incrementos sucesivos, controlando otra sección del algoritmo la marcha en el tiempo de estos incrementos. Al controlar el momento de cada incremento, se tiene cuidado que la acción que se va a adoptar no se interfiera con la más reciente acción, que ha sido ya adoptada. El objetivo es minimizar las perturbaciones en el horno, cuando, por ejemplo, está cambiando de un régimen de producción a otro, o de una velocidad a otra. El algoritmo también ayuda a mantener el peso-litro constante. Examina la velocidad de cambio del peso-litro en el tiempo, y comprueba las diferencias importantes que pueda haber con relación al valor medio deseado, adoptando, cuando ello es necesario, la acción adecuada.

26) Se darán cambios en las variables de la fábrica que no se incluyen actualmente de forma explícita en el algoritmo, y además la fábrica se deteriora en forma acusada entre revisiones generales sucesivas; por ello se han incorporado en el algoritmo métodos para mantener al día los coeficientes y factores constantes de las expresiones básicas. La diferencia entre el punto fijo predicho por el algoritmo y el valor real se recoge constantemente, y cuando esta diferencia sobrepasa un valor prefijado se incorpora en el factor constante. Se pretende actualizar los coeficientes de vez en cuando, a medida que cambian las características internas del horno, a medida que el proceso entra en campos de variabilidad que de otro modo significarían una considerable extrapolación, y a medida que se investigan otras variables. La actualización se consigue con ayuda de programas de análisis regresivo que están almacenados en el computador.

27) La función del segundo algoritmo básico es controlar el flujo de aire secundario. Se registra el contenido de oxígeno de los gases de escape y la temperatura del extremo posterior del horno y se examinan para descubrir valores falsos o estados de alarma; si esto ocurre, al igual que con todos los datos de salida del computador, se registra un mensaje adecuado en uno de los impresores de salida. El objetivo de este algoritmo de control es mantener estas dos variables de la fábrica dentro de ciertos límites. Por ejemplo: si el contenido de oxígeno está fuera de sus límites, y la temperatura a la salida del horno se encuentra dentro de los suyos, el algoritmo calcula una nueva posición del registro de tiro. Se calcula el efecto que se espera en la temperatura del extremo posterior; y si esto tiene como resultado una temperatura que excede los límites, no se toma ninguna acción de control, y el algoritmo calcula el efecto esperado al introducir un cambio en la velocidad del horno, que conduzca a una reducción en el fuel-oil y, por tanto, a una reducción en la temperatura a la salida del horno.

28) Actualmente se está consiguiendo experiencia en la manipulación basada en dos algoritmos y se prevé una serie de avances como consecuencia de este sencillo sistema. 